Pensamiento Crítico N. ${ }^{\circ} 11$, pp. 97-110

\title{
Análisis comparativo del nivel de pobreza y extrema pobreza en la zona norte, región Ancash, caso: Corpanqui y Ticllos
}

Ridberth Ramírez Miranda Adolfo Reyes Escarate Edna Ramírez Miranda

\section{RESUMEN}

En este estudio consideramos la investigación a partir de una demarcación empírica, de la región Ancash, la más alejada y de difícil acceso, en donde las dificultades tanto de localización como las de tránsito son un obstáculo de la lucha contra la pobreza, por lo que la zona de investigación son objetivas y conducen a una investigación cualitativa, de modo que nos condujo a conocer la verdadera situación de la pobreza y la extrema pobreza en Corpanqui y Ticllos.

Palabras clave: Región Ancash, pobreza, extrema pobreza, desigualdad, zonas excluidas, mortalidad y natalidad, nivel de educación.

\begin{abstract}
In this study we consider the research from an empirical delineation of the Ancash region, the most remote and inaccessible, in which the difficulties of both the location and traffic are a major obstacle in the fight against poverty, so the research area are objective
\end{abstract}




\section{Ridberth Ramírez Miranda / Adolfo Reyes Escarate / Edna Ramírez Miranda}

and leads to qualitative research, so that led us to know the real situation of poverty and extreme poverty in Corpanqui and Ticllos.

Keywords: Comparative analysis of poverty and poverty in the area extreme northen Ancash region, case: Corpanqui Ancash region, poverty, extreme poverty, inequality, exclusion zones, mortality, natality level of education.

\section{INTRODUCCIÓN}

El estudio se realizó en el distrito de Corpanqui y Ticllos, ubicada en la sierra de Ancash, en la provincia de Bolognesi, departamento de Ancash; el distrito se encuentra considerado en situación de pobreza extrema, ya que presenta preocupantes indicadores socioeconómicos como la tasa de analfabetismo $(45.1 \%)$, la tasa de mortalidad infantil (67.3\%), la tasa de desnutrición crónica en niños menores de 5 años (80\%), y en donde las familias disponen de menos de un dólar diario para sobrevivir.

La investigación dio la oportunidad de conocer la pobreza y la extrema pobreza y conocer las experiencias, sentimientos y trayectorias de niños, jóvenes, adultos y ancianos, que conviven con la pobreza extrema; son los que sufren con la pobreza sin poder hacer nada. Los hemos escuchado porque no había quién les dé importancia; ni el Estado, ni las organizaciones sociales les han escuchado; son seres abandonados a un destino incierto, con el trauma: "¿Hay para comer hoy día?", porque el mañana no existe. Los hechos se corroboran con 460 fotografías de la zona.

Este conocimiento permitió plantear programas de desarrollo rural, orientados a la generación de oportunidades, y al fortalecimiento y desarrollo de capacidades humanas sustentadas en la generación de valor en las potencialidades de los recursos geográficos, naturales, económicos y sociales, -existentes en Corpanqui-; el caso como ejemplo es el distrito de Ticllos, que ha pasado de una población de 200 a 900 habitantes, por las oportunidades brindadas por la Iglesia Católica.

\section{DISEÑO EXPERIMENTAL, MARCO TEÓRICO O PLANTEAMIENTO DE HI- PÓTESIS}

El trabajo investiga el marco conceptual concerniente al entendimiento y definición de la pobreza extrema por la misma población que vive en esta situación; respuestas al 


\section{Análisis comparativo del nivel de pobreza y extrema pobreza en la zona norte, región Ancash, caso: Corpanqui}

por qué existe la pobreza extrema; propuestas para reducir la pobreza extrema por los mismos pobres; la relación de género y pobreza extrema y la institucionalidad en la vida de los pobres en la sierra del Perú.

Se evalúa el impacto de las estrategias y programas de lucha contra la pobreza rural, desde la óptica de diferentes organismos y de los mismos usuarios, como la Iglesia Católica, ofreciendo propuestas para superar la pobreza extrema sustentadas en las potencialidades susceptibles de creación de valor orientadas a la generación de oportunidades y fortalecimiento de capacidades humanas que concuerdan con las opiniones de reducción de la pobreza y extrema pobreza expuestos por la población de Corpanqui y Ticllos. Se presentan fotografías de ambos distritos.

\section{MARCO TEÓRICO}

Una noción única sobre pobreza y extrema pobreza es una tarea prácticamente imposible. Buena cuenta de ello lo dan la gran cantidad de filósofos, sociólogos, políticos y economistas que a lo largo de la historia han tratado el concepto en todas sus posibles acepciones. Son muchas las cuestiones que se plantean sobre este tema tales como qué significa la pobreza y extrema pobreza (desigualdad).

El concepto de igualdad estaba ligado a la idea de justicia, criterio que permanece aún en nuestros tiempos. Se entendía por justicia el respeto a lo tradicional y a lo jerárquico. Pero poco a poco, el concepto de justicia deja de ser tradicional para pasar a ser más racional al reivindicar la protección de los más débiles frente a la primacía privilegiada de los fuertes o de los pobres frente a los ricos.

En los últimos tiempos hay un renovado interés por la investigación sobre la pobreza y extrema pobreza y por los instrumentos más certeros para su medición. Los estudios por parte de organismos internacionales pertenecientes a la ONU, CEE, sobre crecimiento económico se complementan con análisis sobre el bienestar económico y la equidad distributiva. Ello contribuye a que también en el Perú aumente el interés por esta línea de investigación, si bien una de las limitaciones con que hay que enfrentarse es la escasez de datos reales disponibles para tales estudios. 


\section{Ridberth Ramírez Miranda / Adolfo Reyes Escarate / Edna Ramírez Miranda}

La evolución de los conceptos: justicia social, equidad, pobreza y extrema pobreza, servirán de gran ayuda para comprender mejor las tendencias actuales ante la adopción de medidas de política económica que contribuyan a reforzar el denominado "Estado del bienestar"; no es objeto de nuestra investigación entrar a discernir sobre los diferentes planteamientos filosóficos, sociológicos, o de teoría económica que se dan en la actualidad sobre tales conceptos, sino demostrar su existencia real y contribuir a la medición de la pobreza y extrema pobreza en el Perú.

Las dificultades enfrentadas vienen determinadas por el propio objeto a medir: la pobreza y extrema pobreza. La pobreza y extrema pobreza son conceptos cuyo significado es genérico, pero resulta difícilmente definible. Como se afirma en uno de los informes publicado en el marco del programa de la OCDE, se trata de una idea vaga y abstracta que más bien denota una aspiración que una realidad.

\section{HIPÓTESIS}

La pobreza y extrema pobreza se diferencian en los distritos de Corpanqui y Ticllos por la política aplicada de instituciones benéficas o la intervención del Gobierno.

\section{INDICADORES}

La batería de indicadores sociales del Consejo Económico y Social de las Naciones Unidas, componentes del nivel de vida:

1. Salud

2. Consumo de alimentos y nutrición

3. Educación

4. Empleo y condiciones de trabajo.

5. Vivienda

6. Seguridad social

7. Vestido 
Análisis comparativo del nivel de pobreza y extrema pobreza en la zona norte, región Ancash, caso: Corpanqui

8. Recreo

9. Libertades humanas

\section{Los indicadores sociales en el Perú}

De forma análoga como se hace en los proyectos de la ONU y de la OCDE, se agrupan los temas objetos de investigación en ocho "campos de preocupación social":

\section{Educación}

2. Trabajo

3. Distribución y consumo

4. Protección y servicios sociales

5. Salud

6. Viviendas y medio ambiente

7. Cultura y ocio

8. Oportunidades sociales y participación

Además se prevé la inclusión de tres indicadores adicionales:

9. Población

10. Familias y hogares

11. Investigación y tecnología

\section{MÉTODOS Y RESULTADO}

Se consideró a los distritos de Corpanqui y Ticllos, ubicados en la provincia de Bolognesi, departamento de Ancash, como referentes significativos para el estudio. 


\section{Ridberth Ramírez Miranda / Adolfo Reyes Escarate / Edna Ramírez Miranda}

\section{Método}

Se aplicó el método de Evaluación de la Pobreza con Participación de los Afectados (EPPA), utilizado por el Banco Mundial en estudios de pobreza en más de 100 países del mundo. Este método es un proceso iterativo y participativo que permite entender la pobreza desde el punto de vista de los propios afectados.

Se desarrolló en 10 Talleres Participativos de Planeamiento, focus groups, encuestas aplicadas en Corpanqui y Ticllos; se contó con la participación de hombres y mujeres de diferentes edades; y se realizaron reuniones participativas con los líderes en el distrito: autoridades ediles, representantes de los comuneros, con el propósito de intercambiar experiencias de lucha contra la pobreza realizadas en el ámbito en estudio y el enfoque de desarrollo humano sustentado en el desarrollo de capacidades; se revisaron los diferentes estudios que existen sobre la pobreza rural en América Latina y el Caribe, realizados por diferentes organismos: Banco Mundial, CEPAL, ILPES, FAO, PNUD, FONCODES, Ministerio de Economía y Finanzas, Ex CTAR, INE, entre otros.

Los estudios realizados a la fecha han tratado "la pobreza" en forma general y aplicando métodos diferentes para su medición: necesidades básicas insatisfechas, línea de pobreza, índice de desarrollo humano, índice de pobreza humana. No existen estudios de la pobreza rural en Corpanqui que sean la expresión del mismo pobre y que explique el carácter multidimensional de la pobreza.

De igual manera, los diferentes programas y estrategias aplicados para la reducción de la pobreza se han concentrado en la pobreza en general en áreas urbanas y urbano-marginales y no han tenido incidencia significativa en la reducción de la pobreza extrema en el campo o en la sierra.

\section{Resultados}

Validando estas opiniones de los pobres de la sierra con las informaciones secundarias y opiniones de las personas que viven muchos años en la localidad y de la observación y el análisis podemos apreciar los siguientes hallazgos: en promedio, en Corpanqui los pobres, tanto hombres como mujeres, relacionaron ser pobre con aspectos diversos, como los que se presentan a partir del siguiente esquema: 


\section{Análisis comparativo del nivel de pobreza y extrema pobreza en la zona norte, región Ancash, caso: Corpanqui}

1. Insuficiencia alimentaria: alta tasa de desnutrición crónica. Tiene implicancia directa en el proceso de aprendizaje y la elevada tasa de mortalidad infantil por la desnutrición de las madres gestantes.

2. Desempleo: la actividad agropecuaria responde a su carácter estacional; sin embargo, el desempleo generalmente se observa en el caso de las mujeres que desean trabajar y no encuentran trabajo en su medio, no pueden emigrar por tener mano de obra no calificada y por estar a cargo de sus hijos que en muchos casos son muy pequeños.

3. Subempleo: es una realidad en los hombres que trabajan en las chacras ajenas y en las ciudades, donde reciben un salario por debajo del ingreso mínimo vital y trabajan más de las ocho horas normadas y sin ningún beneficio social.

4. Tierras pobres: predominancia del minifundio (65\%) y tierras en secano (80\%); dependen de las lluvias y están ubicadas en zonas agrestes, erosionadas y de geografía altamente accidentada; se suma la ausencia de capacitación y asistencia técnica.

5. Analfabetismo: la relación entre el no saber leer ni escribir con el ser pobre es estrecha y se relaciona con la elevada tasa de analfabetismo existente en el distrito de Corpanqui.

6. No tener servicios básicos: no cuentan con servicios de agua potable ni mucho menos con servicio de desagüe; se abastecen de agua de los ríos, manantiales y estanques.

7. Abandono familiar: emigran a localidades cercanas para trabajar como peones en la siembra y cosecha de maíz, habas, etc. en procura de ganar ingresos monetarios adicionales para su familia.

8. La migración de la sierra a la costa: para enfrentar la pobreza extrema en que viven y no tienen posibilidad de desarrollo. Los jóvenes son los que mayormente emigran al no tener propiedad alguna que administrar y salen en busca de oportunidades a la costa. 


\section{Ridberth Ramírez Miranda / Adolfo Reyes Escarate / Edna Ramírez Miranda}

9. Alto riesgo de enfermarse: muchos centros poblados se encuentran a más de 15 kilómetros de una posta médica o centro de salud, lo cual se agudiza por la carencia de transporte diario en caso de emergencia; en total, el distrito cuenta con dos postas médicas atendidas por un médico, una enfermera y 4 técnicos en enfermería.

10. Corrupción: tanto hombres como mujeres relacionaron el ser pobre con la gran corrupción de las autoridades y administradores que se han robado la plata de la municipalidad; esta situación hace que la población no confíe en sus autoridades.

11. Educación deficiente: la educación se considera deficiente, siendo una de las razones el bajo nivel de preparación de los docentes (no tienen título profesional de pedagogía), y así no garantizan el proceso eficiente de enseñaza y aprendizaje; se suma la alta tasa de analfabetismo de sus padres que no les pueden apoyar en sus tareas.

Consecuentemente, en el distrito de Ticllos todos los indicadores han variado positivamente. La situación tiende a normalizarse por haber desarrollado actividades de mejoramiento en los ítems indicados a niveles aceptables, es decir, implica una recuperación significativa.

\section{ANÁLISIS Y DISCUSIÓN}

Partiendo del concepto de pobreza como "un síndrome situacional en que se asocian el infraconsumo, la desnutrición, las precarias condicionales de vivienda, los bajos niveles educacionales, las malas condiciones sanitarias", que implican una inserción inestable en el aparato productivo, actitudes de desaliento y anomia, poca participación en los mecanismos de integración social y quizás la adscripción a una escala particular de valores diferenciada en alguna medida a lo establecido como lógica normal de la sociedad; en realidad, se halló situaciones conflictivas difíciles de enfrentar.

En esta investigación se considera que la pobreza y la extrema pobreza son situaciones en que las personas no pueden satisfacer una o más necesidades básicas, por lo tanto no pueden participar plenamente en la sociedad ${ }^{1}$. Relaciona la pobreza con el grado de satisfacción de las llamadas "necesidades básicas", consideradas universales y

1 El mundo de la pobreza; Universidad Mariño; España 2000. 


\section{Análisis comparativo del nivel de pobreza y extrema pobreza en la zona norte, región Ancash, caso: Corpanqui}

que comprenden canasta mínima de consumo individual o familiar (alimentos, vivienda, vestuario, artículos del hogar), el acceso a los servicios básicos (salud y educación, agua potable, recolección de basura, alcantarillado, energía y transporte público) o ambos componentes. Este enfoque explica los diferentes tipos de pobreza en base al acceso que las familias tienen a los diferentes servicios².

\section{DISCUSIÓN}

Como se puede apreciar, la investigación de pobreza y la extrema pobreza no es tarea fácil; no obstante, todo parece apuntar a que la pobreza es una categoría multidimensional y, por lo tanto, no se la puede abordar desde un solo ángulo, sino que debe ser planteada como un problema complejo que involucra factores de índole económica, social, cultural, moral, política e incluso natural; definida con la participación y visión de los entendidos en la pobreza, vale decir, los mismos pobres, en el análisis de su propia condición de sobrevivencia; eso se logró en la presente investigación, luego de una ardua contrastación de sus posiciones. Se asumió el concepto de los pobres que definieron la pobreza como:

- No tener nada para que coman sus hijos.

- Ser golpeados y vejados siempre.

- Morirse por no tener medicamentos.

- No ir a la escuela, no saber leer ni escribir.

- Trabajar gratis y si se trabaja pagan poco.

- Beber agua contaminada.

- Indiferencia y maltrato de las autoridades y funcionarios corruptos.

- Inexistencia total del Poder Judicial, porque no hay justicia para los pobres.

$\overline{2}$ La pobreza y el desarrollo humano. 


\section{Ridberth Ramírez Miranda / Adolfo Reyes Escarate / Edna Ramírez Miranda}

A su entender, la pobreza "Es una enfermedad maligna, que no tiene cura"; "Es un dolor profundo, que hace doler el corazón"; "Es su destino de ser pobre"; "Es morir poquito a poquito, todos los días"; "Es seguir siendo pobre igual que sus padres".

\section{CONCLUSIONES}

1. La pobreza y extrema pobreza en Corpanqui es de carácter permanente y generacional, por lo que urge cambiar las estrategias de lucha contra la pobreza. Los programas no son suficientes por cuanto homogenizan su accionar; el problema es heterogéneo e implica estrategias ajustadas a su realidad.

2. La pobreza y extrema pobreza forman un círculo vicioso. Para revertirlo se requiere disminuir las diversas causas mencionadas por los mismos pobres; ello puede ser posible a través de la generación de oportunidades de desarrollo humano sostenible aprovechando las potencialidades existentes en su medio.

3. Enfrentar la pobreza y extrema pobreza implica convertir las ventajas comparativas en ventajas competitivas a través de la calidad educativa en los niveles inicial, primario, secundario y universitario. Apuntar a la erradicación total del analfabetismo.

4. Promover inversiones en infraestructura básica, para incrementar la producción y productividad de los factores económicos.

5. En el distrito de Ticllos, el sacerdote ha logrado elevar el nivel educativo recreando las escuelas inicial, primaria, y secundaria, además de fundar institutos tecnológicos como los de carpintería, ebanistería, tallado, cultivo de tierras, producción de abonos, producción de leche, etc.; la población lo va logrando como se puede observar en las fotografías tomadas in situ.

6. Se adjuntan fotografías tomadas de los dos distritos: Corpanqui y Ticllos. Se considera que las fotografías son testimonio de lo que ocurre realmente en estos distritos que se ha investigado; se puede observar la realidad concreta de la situación de pobreza y extrema pobreza en que se encuentran. 


\section{Análisis comparativo del nivel de pobreza y extrema pobreza en la zona norte, región Ancash, caso: Corpanqui}

\section{BIBLIOGRAFÍA}

Antezana A. Julián. Dinámica demográfica y pobreza en el Perú. Edic.; CELADE; Perú, 1997; p. 15.

Arriagada, Irma. Dimensiones de la pobreza y políticas desde una perspectiva de género. Edic. 1 ONU.; Chile; 2005; p. 176.

Ballen, Mariana. Una evaluación de los programas de lucha contra la pobreza rural en el Perú: FONCODES y PRONAMACHS; FONCODES; Perú; 2001; p. 86.

Banco Mundial. "Informe sobre el desarrollo mundial 1991. La tarea acuciante del Desarrollo". Washington, 1991; p. 58.

Boltvink, J. Pobreza y necesidades básicas. Edic.1; ONU; Chile; p. 185.

Caldera, Andrés y Jarquin, Edmundo. Programas sociales, pobreza y participación ciudadana; B.M; México; 2005; p.132.

Chant, Sylvia. Nuevas contribuciones al análisis de la pobreza. ONU; Chile; 2003; p. 80.

Coben, Ernesto y Franco, Rolando. Gestión social: cómo lograr eficiencia e impacto en las políticas sociales. Siglo XIX; México; 2005; p. 316.

Cotler, Julio. Desarrollo campesino en los Andes, cambios tecnológicos y transformación social en las comunidades campesinas del Perú. IEP; Perú; 1989; p. 67.

Cuadrado Roura, J.R. "Tendencias económico-regionales antes y después de la crisis en El Perú”. Papeles de economía, №34, 1988; pp. 17-61.

Deepa, Narayan. La pobreza es impotencia y falta de representación. F.D; Perú; 2000; p. 30. 


\section{Ridberth Ramírez Miranda / Adolfo Reyes Escarate / Edna Ramírez Miranda}

Ebert, U. "Family of aggregative compromise INE quality measures". International Economic Review. Vol. 29, N. ${ }^{\circ}$ 2, may 1988; pp. 363-376.

Echart, Enara. Globalización, pobreza y desarrollo. AGAPEA; España; 2003; p. 448.

El Mundo de la pobreza. Universidad Mariño; España, 2000.

Escobar, Javier y otros. Los activos de los pobres en el Perú. GRADE; Perú; 1998; p. 89.

Feres, Juan Carlos. Sobre el método de medición de la pobreza. CELADE; Perú; 1998; p. 20.

Figueroa, Adolfo. La cuestión distributiva en el Perú. IEP; Perú; 1998; p. 60.

Fritz, Du y Shack, Nelson. Balance y perspectiva en la lucha contra la pobreza. BM; Perú; 1999; p. 120.

Godoy, Lorena. Entender la pobreza desde la perspectiva de género. ONU, Chile; 2004; p. 128.

Kaztman, Rubén. La medición de las necesidades básicas. CELADE; Perú; 1998; p. 35.

Luistig, Nova. Reducción de la pobreza y desigualdad. BID; Estados Unidos; 2001; p. 30.

Marín, Juan Carlos. Instrumentos de programación, focalización y evaluación de políticas sociales. CELADE; Perú; 1998; p. 20.

Martínez P., Jorge. Algunas interrelaciones entre pobreza y dinámica demográfica. CELADE; Perú; 1998; p. 24.

Morley, Samuel. Estrategias para reducir la pobreza. BID; Estados Unidos; 1997; p. 108. 


\section{Análisis comparativo del nivel de pobreza y extrema pobreza en la zona norte, región Ancash, caso: Corpanqui}

O.C.D.E. "La liste des indicateurs sociaux", 1982. Traducción española publicada por el Ministerio de Trabajo y Seguridad Social: "Indicadores sociales lista O.C.D.E." Madrid, 1985; p. 5.

O.C.D.E. "Les conditions de vie dans les pays de L'OCDE. Recueil d'Indicateurs Sociaux", Paris, 1986.

O.C.D.E. "Measure du bien-étre social. Progres accomplis dans l'elaboration des indicateurs sociaux". Paris, 1976. Traducción española del I.N.E. Perú, 1981; p. 21.

ONU. "Hacia un sistema de Estadísticas..."; p. 4

ONU. "International definition and measurement of levels of living". E/CN.3/270.9 de mayo de 1960. Referencia en Pena Trapero: o.c.; p. 22.

Olavartia Mauricio. Pobreza; ONU; Chile; 2001; p. 130.

ONU. "Indicadores sociales: directrices preliminares y series ilustrativas". Informe Estadístico ST/ESA/STAT/SER.M/63. Nueva York, 1978.

Ortiz de Cevallos, Gabriel y otros. Lucha contra la pobreza. IEP; Perú; 200; p. 85.

Pena Trapero, J.B. "Indicadores socioeconómicos". Art. cit.; p. 260.

Reyes M., Jorge. Presentación de metodologías aplicadas en países. Edic.1; CELADE; Perú; 1998; p. 20.

Romero, Alberto. Globalización y pobreza. Libro Net; Estados Unidos; 2002; p. 200.

Sen, Amartya K. Desarrollo y libertad. España; 2000; p. 65.

Velazco Portocarrero, Jackeline. Las actividades rurales no agrícolas en las familias campesinas de la sierra norte del Perú. FAO; Perú; 2003; p. 30. 


\section{Ridberth Ramírez Miranda / Adolfo Reyes Escarate / Edna Ramírez Miranda}

Viveros, Alberto. Programas de reducción de la pobreza en América Latina. N.U. Chile; 2001; p. 64.

Webb, Richard. Pobreza en el Perú: estimaciones y estrategias. IEP; Perú; 1985; p. 67.

Webb, Richard. Políticas gubernamentales y distribución del ingreso en el Perú 19631973. IEP; Perú; 1978; p. 78.

Yamada, Gustavo, Jarque y otros. Reducción de la pobreza y promoción de la equidad social; BID; Estados Unidos; 2003; p. 46. 